\title{
El tratamiento empírico en jóvenes con dispepsia ulcerosa y Helicobacter Pylori fue mejor que el basado en el manejo endoscópico
}

A prospective randomized trial of a "test and treat" policy versus endoscopy based management in young Helicobacter pylori positive patients with ulcer-like dyspepsia, referred to a hospital clinic. Heaney A., Collins J, Watson R, Mc Farland R. Gut 1999; 45:186.

\section{Objetivo}

Comparar dos estrategias diferentes de manejo de la dispepsia ulcerosa en pacientes jóvenes con Helicobacter pilory (HP): tratamiento empírico o un manejo basado en endoscopía.

\section{Diseño}

Estudio prospectivo, aleatorizado y estratificado* según sexo, tabaquismo e ingesta de alcohol.

\section{Lugar}

Centros de gastroenterología de dos hospitales en Irlanda del Norte.

\section{Pacientes}

Ciento cuatro pacientes (edad media 32 años, 57\% hombres) incorporados de manera consecutiva, derivados para realización de endoscopía. Criterios de inclusión: menores de 46 años con dispepsia ulcerosa y con HP positivo evaluado a través del test de la urea espirada marcada con C13. Criterios de exclusión: síntomas de alarma: (pérdida de peso, disfagia), síntomas de reflujo gastroesofágico, historia de sangrado duodenal, uso regular de antiinflamatorios, sintomas sugestivos de litiasis vesicular, embarazo, tratamiento de erradicación del HP en las dos semanas previas.

\author{
Intervención \\ Los pacientes fueron randomizados para recibir tratamiento \\ empírico ( $n=52)$ o un manejo según el resultado de una endos- \\ copía $(n=52)$. Seguimiento a doce meses.
}

\section{Medición de Resultados Principales}

Se evaluaron la severidad de la dispepsia, a través de un puntaje (Glasgow dyspepsia severity score); y la calidad de vida relacionada a la salud a través del cuestionario SF36.

\section{Resultados}

Fueron analizados los grupos por intención de tratar*. Los puntajes de dispepsia mejoraron en ambos grupos con respecto del basal, pero los síntomas fueron menores en el grupo de tratamiento empírico: Puntaje de Glasgow $=3,37$ vs. 5,08; $p<0,05$. $E \mid$ score de calidad de vida mejoró en ambos grupos a los doce meses. La funcionalidad física mejoró significativamente más en el grupo sometido a tratamiento empírico: $\mathrm{SF} 36=91,88$ vs. $81,96, p<0,05$. El $27 \%$ de los pacientes en el grupo de tratamiento empírico recibieron endoscopía por no mejorar sus síntomas.

\section{Conclusiones}

Este estudio avala la erradicación del HP con la estrategia de testear y tratar. Se estima que un $73 \%$ de endoscopías se evitarían con esta estrategia, sin detrimento en los resultados.

\section{Comentario}

La infección por HP es una de las infecciones crónicas más frecuentes 1 . En los pacientes con dispepsia HP positivos, el $40 \%$ tienen úlcera duodenal, el $13 \%$ gástrica, mientras que de los pacientes HP negativos, sólo el $2 \%$ tienen úlcera duodenal, y el $3 \%$ gástrica 2 . El tratamiento erradicativo del HP ha demostrado curar la enfermedad ulcerosa péptica (EUP); pero no está indicado en la dispepsia no ulcerosa con la evidencia actual 3 . El test de urea espirada, aunque no ampliamente disponible, es en la actualidad el standard diagnóstico tanto para la detección como para evaluar la eficacia en el tratamiento erradicativo del HP. La sensibilidad y especificidad en sujetos no tratados va desde el $90-98 \%$ y el $92-100 \%$, respectivamente 2,4 . Los otros test diagnósticos (biopsia, serología, CLO-test) tienen un rédito algo menor, pero se hallan más disponibles. Las estrategias de manejo la EUP van desde el tratamiento empírico con antiácidos hasta el estudio endoscópico precoz. El presente estudio trata de demostrar la utilidad de la detección del HP en pacientes con dispepsia sin síntomas de alarma y tratamiento empírico a los positivos (grupo A), comparándola con el manejo basado en los hallazgos endoscópicos (grupo B). Concluye que ambas estrate- gias son útiles para disminuir los síntomas, favoreciendo al año al grupo A, según el puntaje de dispepsia y el de calidad de vida, en el funcionamiento físico (sí bien en este último ítem el puntaje inicial es significativamente mejor en la rama de tratamiento empírico). Una limitación del estudio, reconocida por los autores, es la presunción de que los pacientes tengan la misma patología en ambos grupos. Más visible en términos clínicos es que en el período de 12 meses se evitaron un $73 \%$ de endoscopías en el grupo A. Así mismo, el $43 \%$ de los pacientes en el grupo A y el $30 \%$ de los del grupo B estaban asintomáticos, y el $24 \%$ vs. el $37 \%$ de los pacientes del primer y segundo grupo, respectivamente, tomaban anti $\mathrm{H} 2$ o inhibidores de la bomba de protones. Este estudio sugiere que la estrategia de testear y tratar es comparable a la estrategia más invasiva y costosa de realizar endoscopía temprana a los pacientes jóvenes con dispepsia. Interrogantes pendientes de aclaración en el manejo de la dispepsia son a quiénes realizar estudios no invasivos para la detección del HP y cuáles son los potenciales beneficios y perjuicios del tratamiento masivo contra HP en los pacientes con dispepsia.

\section{Dr. Javier Pollán [ Servicio de Clínica Médica. Hospital Italiano de Buenos Aires ]}

\author{
Referencias \\ 1. Rabeneck L, Graham D. Helicobacter pylori: When To Test, When To Treat. Ann Intern Med. 1997 Feb 126: 315-316 \\ 2. Talley N, Axon A, Bytzer P y col. Management of uninvestigated and funtional dyspepsia: a working party report for the World Congresses of Gastrenterology 1998 . Aliment Pharmacol \\ Ther. 1999 Sept 13(9): 1135-1148 \\ 3. Talley N. How should Helicobacter pylori positive dyspeptic patients be managed? Gut. 1999 July 45 suppl I: 128-131 \\ 4. Savarino V, Vigneri S, Celle G. The 13C urea breath test in the diagnosis of Helicobacter pylori infection. Gut. 1999 July 45 suppl I: 118-122
}

